\title{
Diskurs und Erkenntnis: Ein (nicht ganz) fiktiver Dialog zwischen T. Singelnstein (ts) und D. Fabricius (df)
}

Die folgende Buchbesprechung führte zu einem intensiven E-Mail- und persönlichem Austausch zwischen Buch- und Besprechungsautor. Die wesentlichen Elemente dieses Austausches haben wir aufgenommen und in die Form eines - beide zufrieden stellenden - Dialoges gebracht, der im Anschluss an die Besprechung zu finden ist.

\section{Besprechung (Dirk Fabricius)}

Singelnstein, T. (2009), Diskurs und Kriminalität. Außergesetzliche Anwendungsregeln als diskursive Praktiken im Wechselverhältnis zwischen Kriminalisierungsdiskursen und Strafrechtsanwendung. Berlin: Duncker \& Humblot, 227 S., 78,- $€$.

Das gesellschaftskritische Paradigma in der Kriminologie, das sich im vergangenen Jahrhundert zunächst im Angloamerikanischen und in den 1960er und 1970er Jahren auch in Deutschland entwickelt und etabliert hat, war und ist stark von konstruktivistischen Ansätzen verschiedener Couleur geprägt. Insbesondere der labeling approach bzw. Etikettierungsansatz griff die vorherrschende kriminologische Perspektive an, in der Kriminalität und Kriminelle als feststehende Kategorien erscheinen, und machte demgegenüber die gesellschaftlichen Zuschreibungsprozesse zum Thema, die diese Kategorien produzieren. Dieser Perspektivenwechsel hat die deutsche Kriminologie nachhaltig beeinflusst. Neuere sozialwissenschaftliche Ansätze im interpretativen Paradigma auf ihren Nutzen für kriminologische Fragestellungen hin zu untersuchen, scheint daher vielversprechend.

Viel diskutiert wird die Diskursanalyse bzw. Diskursforschung, bei der es sich um ein schillerndes Feld ganz verschiedener Ansätze handelt. Gemein ist diesen, dass sie Diskurse als gesellschaftliches Phänomen auffassen, das es in Entstehung, Verlauf und Wirkung zu untersuchen gilt. Unterschiede zeigen sich bereits beim Diskursbegriff, dem disziplinären Hintergrund und dem methodischen Herangehen. In diesem Sinne lassen sich grob eine sprachwissenschaftlich orientierte, eine historische, eine ideologiekritische sowie eine sozialwissenschaftlich-wissenssoziologische Richtung unterscheiden. Ziel der Arbeit ist es, diese in den Sozialwissenschaften bereits sehr ausgeprägte Forschungsrichtung im Anschluss an bisherige Ansätze im gesellschaftskritischen Paradigma für kriminologische Fragestellungen fruchtbar zu machen.

Im Zentrum der Arbeit steht eine umrisshafte Analyse von Diskursen zu Delikten mit extrem rechtem Hintergrund und deren strafjustizieller Bearbeitung (Kapitel E, S. 114 ff.). Das zentrale Kapitel E belegt, dass die Strafrechtsanwendung sich unter den wechselnden Einflüssen eines allgemeineren gesellschaftlichen und zweier benannter speziellerer Diskurse, des juristischen und kriminologischen, wandelte. Die allgemeinere Einschätzung von Brandstiftungen und gewalttätigen Angriffen beeinflusste Entscheidungen in speziellen Fällen. Delikte mit extrem rechtem Hintergrund, das sind die mit „Rostock-Lichtenhagen“, „Mölln“ und „Solingen“ exemplarisch benannten. Die 
Modell-Täter wandeln sich von alkoholisierten, beschädigten Einzeltätern zu organisierten (in des Wortes doppelter Bedeutung), gelten damit als gefährlicher. Der individuelle Täter wird tendenziell dem Modell entsprechend eingeschätzt. Ebenso wird eher Tötungsvorsatz angenommen, z.B. bei Einsatz bestimmter Schlagstöcke. In der Tat: außergesetzliche Anwendungsregeln als diskursive Praktiken. Kriminalisierungsdiskurse, die die Strafrechtsanwendung bestimmen (S. 147-153): „Delikte mit extrem rechtem Hintergrund“. Beobachtungen und Handlungen allerdings brechen hier ,außerdiskurslich" ein - was der theoretische Rahmen, den Singelnstein schafft, nicht vorsieht und was, wie ich zeigen möchte, diese Theorie sehr problematisch macht.

Zunächst verortet der Autor seinen diskursanalytischen Ansatz, wendet sich der bisherigen Instanzenforschung zu (B, S. 20 ff.), betrachtet Rechtsanwendung im Prozess der Kriminalisierung (C, S. 39 ff.) und geht speziell auf die Diskursanalyse (D, S. 67 ff.) ein. Das letzte Kapitel (F, S. 167 ff.) beschäftigt sich mit Macht in der Foucaultschen Tradition. So entsteht fast ein Lehrbuch der Diskurstheorie. Ich werde herausarbeiten, wie die Diskurstheorie danach Diskurs, Diskursanalyse und spezieller Kriminalität und Recht versteht, sind dies doch die zentralen Termini des Titels. Das zentrale Problem mein zentrales Problem? - wird offenbar, wenn man fragt, was Wissen und Wirklichkeit i.S.d. Diskurstheorie sind. Beim Nachdenken über eine passende Überschrift für diese Besprechung kamen mir Einfälle wie „Der Fluch der Geisteswissenschaft“ oder „Im Diskurs verloren“. Denn die Theorie derealisiert Wirklichkeit. Wissen wird damit unmöglich. Singelnstein entgleitet, wenn er sich dieser Theorie ohne Wenn und Aber verschreibt, die Möglichkeit, gesellschaftliche Realität jenseits eines Diskurses für wahrnehmbar zu halten, jedenfalls kann er sie nur noch implizit und indirekt anführen. Abschließend gehe ich noch auf die „Machtfrage“ ein, die unbefriedigend bleibt, weil sie in dem Diskurs hängen bleibt, der es verbietet, bestimmte Fragen überhaupt noch zu stellen.

\section{Diskurs}

Als Diskurs werden ,strukturierte, sich wandelnde überindividuelle Wissensvorräte verstanden, die im Zusammenspiel mit nicht-diskursiven Praktiken in Form von Handlungen, Sichtbarkeiten und Vergegenständlichung soziale Wirklichkeit konstituieren und so das Handeln und Denken der Subjekte leiten." (S. 14; s. auch S. 61, 67, 79).

Diskurse konstituieren soziale Wirklichkeit (S. 14, 26, 90, 92). „Ohne diskursive Rezeption gibt es aus Sicht der Diskursanalyse ein Ereignis nicht.“ (S. 93). Entsprechend „richtet sich die Fragestellung der Diskursanalyse nicht darauf, ob ein bestimmtes Geschehen materiell stattgefunden hat. Betrachtet wird vielmehr umgekehrt die Frage, wie sich ein bestimmtes Wissen als Wahrheit herausbilden konnte, wie es Wirklichkeit produziert, indem es sich in die Praktiken der Subjekte niederschlägt und dort umgesetzt wird.“(S. 90). „Aus der Sicht der Diskursanalyse“: Würde diese Grenze durchgehalten, wäre die Diskursanalyse nützliches Instrument, sich auf ein bestimmtes „Operationsfeld“, auf welchem man mit begrenzten Mitteln unterwegs ist, zu beschränken, ohne zu übersehen, dass eine solche Analyse nur ein „,verschwindendes Moment“ im Kontext einer Kriminalitätstheorie sein kann. Der Chirurg, der vergäße, dass die postoperativen 
Konsequenzen seiner Schnitte körperliche und seelische Gesundheit und Wohlbefinden der Patienten beeinflussen, wäre kein guter Arzt.

Diskursives Wissen bildet eine überindividuelle Wissensebene, welche der Kontrolle der Subjekte entzogen ist (S. 73, 75). Etwas anders liest sich das auf S. 77, wonach „Diskurse... keine einseitig wirkmächtige vom Handeln der Subjekte unabhängige Struktur darstellen," sondern mit dieser in einem Austausch stehen. Dennoch ist diese Wechselwirkung schwach. Der Wissensrahmen entsteht strukturell, jenseits intentionalen Handelns, und nur innerhalb dieses Wissensrahmens ist intentionales Handeln möglich. „Das diskursive Wissen geht sozialer Wirklichkeit... voraus und bestimmt diese." (S. 200).

Entsprechend legt das diskursive Wissen fest, ,welche Verhaltensweisen unter welchen Umständen Kriminalität darstellen oder nicht.“ (S. 94) „Das Wissen der Diskurse... schlägt sich auch in Materialisierung nieder." (S. 94) Was heißt Materialisierung? Eine Materialisierung sei auch das Recht, als eine Verkörperung der gesellschaftlichen Wahrheit (S. 94 f.). Materialisierung führt nicht zu Materialität, denn die materiellen Gegenstände sollen ausgeschlossen werden - das wird auf S. 31 bekräftigt. Obwohl oft verwendet, erhellt sich mir der Sinn nicht.

Nachdem den Diskursen so viel Macht zugesprochen wird, fragt man sich, ob es Diskurse gibt oder ob sie erst durch die Benennung im Kontext eines Diskurses existent werden. Das bleibt unbeantwortet.

\section{Diskursanalyse}

Die Diskursanalyse soll „,den herrschenden kulturellen Horizont auf Distanz“ bringen und hinterfragen (S. 14). Es soll herausgefunden werden, wie ,im Zuschreibungsprozess Wertungen vorgenommen werden" und auch, wie sie sich konstituieren und durchsetzen. Das wird mit einer Machtanalytik verbunden (S. 17).

Die Diskursanalyse wird dem interpretativen Paradigma zugeordnet (S. 21). Es gebe keinerlei soziale Strukturen außerhalb der Interpretation, denn soziale Strukturen kämen durch individuelles Verhalten zustande, das wiederum sei an Bedeutungen geknüpft. Diese Bedeutungen resultierten aus der Interaktion (S. 21 ff.). Sachverhaltsfeststellungen können danach nur als eine von vielen möglichen Interpretationen verstanden werden (S. 42), es gehe in Kriminalisierungsprozessen „nicht um die Beurteilung einer feststehenden Wirklichkeit, sondern um die Interpretation eines Geschehens als Sinnzuschreibung, die bereits in die Wahrnehmung eines Geschehensablaufs hineinwirkt und diese prägt.“ (S. 43). Dieser Begriff von sozialer Realität setzt die Trennbarkeit von Materie und Idee (S. 22, 26, 79) voraus. „Gesellschaftliche Gegebenheiten werden... als äußerlich vorhanden und zwingend wahrgenommen, sie werden jedoch nur durch die permanente Umsetzung und Behandlung im Alltag real.“ (S. 23). Ich wende ein: Sachverhalte, wörtlich, sind Relationen zwischen Sachen. Relationen sind abstrakt und immateriell. Gesellschaft lässt sich am besten als Inbegriff von Beziehungen zwischen Positionen im sozialen Raum bestimmen. Im Prinzip lässt sich dies für einen gegebenen Zeitpunkt feststellen. Die kategoriale Trennung von Materie und Idee, Stoffwechsel und Informationswechsel ist sinnvoll. Jedoch sollte man abstrakte Gegenstände nicht für irreal oder nicht feststellbar halten. Und nie übersehen, dass die Relationen zwischen 
Positionen bestehen, die tatsächlich materielle Aspekte haben. Der Sozialwissenschaftler kann die realen Strukturen mehr oder minder richtig erkennen. Seine Voraussagen können mehr oder weniger zutreffen. Im ,interpretativen Paradigma“ kann man den „herrschenden kulturellen Horizont“ nur aus einem Meta-Diskurs heraus ,auf Distanz bringen", der jedoch nicht Wahrheit, sondern machtvolle Interpretation als Geltungsgrund haben muss. Schon in der Kopernikanischen Wende griffen astronomische Erkenntnisse über Materielles (Erde, Sonne, Planeten) und ihre Relationen das herrschende Weltbild an. Dessen Vertreter suchten mit den Mitteln der Inquisition diese Erkenntnisse auszulöschen. Ich neige zu der Annahme, dass das heliozentrische Weltbild, unabhängig von jedem Diskurs, wahrer als das geozentrische ist.

\section{Kriminalität und Recht}

Kriminalität, so heißt es, werde ,,als soziale Wirklichkeit durch Bestände gesellschaftlichen Wissens konstituiert“" (S. 113). Dieser Prozess wird Kriminalisierung genannt, er kommt durch wertende Zuschreibung zustande und daher ist Kriminalität ein ,soziales Konstrukt“ (S. 13). Kriminalität bildet eine „Sinnprovinz“ (S. 65, 73). Singelnstein strukturiert den Kriminalitätsbegriff durch die Dichotomie normal/abweichend (S. 16) und bezeichnet als abweichend, was im Widerspruch zu herrschenden sozialen Verhaltensanforderungen steht (S. 28, 35). Die Existenz von Kriminalität wird trotz dieser kontingenten Bestimmung ebenso als selbstverständlich wahrgenommen wie Strafe als notwendig angesehen (S. 99). Für diese Kriminalisierung ist nicht in erster Linie der Gesetzgeber zuständig, sondern die Instanzen der sozialen Kontrolle füllen den Kriminalitätsbegriff durch offene und verdeckte Wertungen und Entscheidungen aus (S. 14).

Recht sei weniger als feststehende Hierarchie aus Norm und Rechtsquellen zu begreifen, sondern als „dynamische Vielfalt von Akteuren, Apparaten und Systemen“, das sich beständig selbst reproduziere (S. 15). Als Funktion von Recht wird allein Stetigkeit benannt (S. 64). Recht und Gesetz werden nicht als Konstitutiva sondern ,als selbst von anderen Ebenen bestimmt angesehen.“ (S. 95). Leider erfährt man darüber wenig. Kurz: Recht bleibt durch die Arbeit hindurch seltsam unterbelichtet.

\section{Wissen und Wirklichkeit}

„Diskurse [beschreiben] nicht Realität, sondern konstituieren sie vielmehr, indem sie Wissen, Bedeutung und Sinn hervorbringen, verbreiten, als wirklich und wahr darstellen. Auf diesem Wege bringen sie eine symbolische soziale Ordnung ebenso wie die Subjekte selbst hervor.“ (S. 26). Ersichtlich hat der Diskurs eine umfassende schöpferische Kraft, ein Erster Beweger. Dementsprechend heißt es dann auch: „Insbesondere geht er [sc. dieser Ansatz] nicht davon aus, dass es eine objektive, erfahrbare Wahrheit gäbe, die nur durch die ,falsche“ Wahrheit des Diskurses verdeckt würde. Vielmehr postuliert sie, dass sich die Dinge selbst, das tatsächliche, reale Substrat der Materialität in keiner Weise von sinnhafter Zuschreibung trennen und befreit darstellen lassen. Unsere Wirklichkeit ist danach immer bereits eine Interpretation der Materialität bzw. basiert Wirklichkeit gar selbst auf diskursivem Wissen, so dass es eine ,tatsächliche‘ objektive Wahrheit nicht geben kann. Dementsprechend geht es diesem diskursanalyti- 
schen Ansatz nicht um die Freilegung einer verdeckten Wahrheit, sondern um die Darstellung der Kontingenz von Wirklichkeit, d.h. darum zu zeigen, dass diese nicht festgelegt ist, sondern auch anders sein könnte, werden kann, bloße Möglichkeit ist." (S. 26).

Einschränkend heißt es später: „Damit negiert diese Perspektive weder soziale Wirklichkeit, noch erklärt sie diese für beliebig, wie relativistische oder vergleichbare konstruktivistische Ansätze dies tun. Sie geht vielmehr nur davon aus, dass soziale Wirklichkeit nicht objektiv feststehend ist, sondern auf gesellschaftlichem Wissen basiert und durch dieses erst hervorgebracht wird.“"(S. 201).

Der Schöpfer bleibt ungenannt. Doch wenn soziale Wirklichkeit nur auf Wissen basiert, Wissen wiederum vom Diskurs erzeugt wird, kann der Hervorbringer nur der Diskurs sein. Das impliziert wiederum, dass man Wissen von Irrtum oder Unwissen nicht mehr unterscheiden kann. Denn da der Diskurs sich der Frage der Realität entzieht, es nicht um die Wahrheit der Aussagen geht, die der Diskurs explizit oder implizit über Realität macht, sondern diese Aussagen unmittelbar als Wissen gelten, ist eine Prüfung dieses diskursiven Wissens gänzlich unmöglich geworden. Subjektive und objektive, subjektivierte und objektivierte Wirklichkeit werden kontrastiert, ohne dass jedoch ein Begriff derselben erarbeitet wird. Diese Position muss sich mit einer Wendung gegen ontologische, naturalistische und positivistische Ansätze verbinden - sonst wäre sie nicht durchhaltbar (vgl. S. 31, 33, 127).

Wie sich noch zeigen wird, ist Singelnstein zu klug, um sich dem vollkommen zu verschreiben. Bedauerlicherweise gibt er jedoch mit dieser Verhaftung an ein Paradigma der Interpretation die Möglichkeit preis, seine durchaus robusten Realitätswahrnehmungen theoretisch zu platzieren.

\section{Macht}

„Bereits in seinen früheren Arbeiten hatte Foucault Macht nicht im herkömmlichen Sinne und vordergründig als Herrschaft verstanden, sondern vielmehr im Gegensatz hierzu eine Ebene der ,Mikrophysik der Macht' konzipiert, die er von der von ihm so bezeichneten juridischen oder souveränen Macht abgrenzte. Die Ebene der Macht verstand er als vielfältige, organisierende Kräfteverhältnisse und bewegliche Strategien, die allgegenwärtig sind, von unten nach oben verlaufend alles durchdringen und deren Herausbildung mit dem Aufkommen der Bevölkerung als Entität und möglichem Objekt der Einwirkung in Verbindung steht.“ (S. 172). Diese zum Beginn des entsprechenden Kapitels gegebene Passage bleibt blass. An anderer Stelle (S. 169) wird „Kriminalisierung als Auseinandersetzung um gesellschaftliche Ressourcen“ verstanden. Jedoch bleiben diese gesellschaftlichen Ressourcen ebenso unbestimmt wie die Frage, wer sich von der Bevölkerung getrennt hat und auf sie einwirken kann, noch wie oben und unten zustande kommen.

In der „Zusammenschau“ (S. 196 ff.) heißt es: „Die Rechtsanwendung bei der Kriminalisierung lässt sich als von Regierungstechniken im Sinne der Gouvernementalität durchzogen beschreiben. Fremd- und Selbstführung begegnen sich und interagieren hier in zweifacher Weise: Ebenso wie sich im diskursiven Wissen eine bestimmte Interpretation machtvoll durchgesetzt hat, wirkt sie ihrerseits für den Einzelnen leitend und motivierend." (S. 196) Befremdlich mutet an, dass Singelnstein die Instanzen des Kri- 
minalisierungsprozesses beschreibt als „Komplexe aus sozialen Normen..., die von einer Mehrzahl von Personen umgesetzt werden zur arbeitsteiligen, rationalen Erreichung eines formalisierten Ziels." (S. 62) Die Frage taucht auf, wie dieses rational formulierte Ziel beschrieben werden kann, wenn doch diese Instanzen den Diskurs aktuell hervorbringen bzw. von ihm getragen sind. Eine rationale Erreichung dieses Ziels ließe sich nur feststellen, wenn das Ziel metadiskursiv zu bestimmen wäre.

An anderer Stelle heißt es, „Diskurse [seien] nicht als intentionale Herrschaft, sondern als Struktur zu begreifen, die aufgrund vielfältiger Bedingungen und Wechselverhältnisse mit sozialen Praktiken steht und sich wandelt.“" (S. 77). Diskurse also als nicht intentionale Herrschaft? Aber wer herrscht? Sowohl der Macht- wie der Herrschaftsbegriff bleiben verschwommen und man wird auf den Diskurs als den Schöpfer von gesellschaftlicher Wirklichkeit und von Subjekten zurückgeworfen.

Eine derartige Beschreibung von Macht- und Herrschaftsverhältnissen ist, so möchte ich behaupten, Resultat der seltsamen Trennung von sozialer und physischer Realität und der Preisgabe eines Begriffs sozialer, gesellschaftlicher Realität jenseits eines wirkmächtigen Diskurses.

Sobald man auch physische Ressourcen im Blick hat, entsteht unausweichlich die Frage, wer denn für die Instanzen sozialer Kontrolle, für Gerichtsgebäude, Gefängnisgebäude, für die Ausbildung und Anstellung des Personals etc. bezahlt und warum.

\section{Indiskursive Realität}

In seinem zentralen Kapitel über die Analyse rechter Gewalt beschäftigt sich Singelnstein damit, wie Ereignisse um brennende Asylbewerberheime und dabei zu Tode gekommene Menschen verarbeitet werden. Die Rekonstruktion der Verarbeitung dieser Ereignisse im diskursanalytischen Rahmen macht die These jedenfalls plausibel, dass die Rechtsanwendung, sowohl hinsichtlich der Sachverhaltsfeststellungen wie auch der rechtlichen Bewertung, durch Vorgänge innerhalb der Instanzen sozialer Kontrolle bestimmt ist und dass es hier nicht die individuellen Personen in Interaktionen sind, sondern dass es eine überindividuelle (freilich von Individuen nicht zu trennende) Ebene gibt.

Allerdings führt Singelnstein die genannten Ereignisse ein. Er nimmt „diese Entwicklung“" (S. 149, 150), er sieht Ereignisse als gegeben an, wie auch Tote (S. 150). Es gibt Beobachtungen (S. 155) und einschlägige Geschehensabläufe (S. 161). Es gibt Wissensbestände (S. 151), die wieder verloren gehen (S. 153).

Der Status dieser Entwicklungen, Ereignisse und des Wissens über Geschehensabläufe muss jedoch bei der erkenntnistheoretischen Basis zweifelhaft bleiben. Sie können nur als ,hereingeschmuggelt“ angesehen werden. Wenn man diese Entwicklungen für beobachtbar hält und annimmt, dass man darüber Wissen haben, aber auch verlieren kann, so ist der Diskurs in seiner generativen Kraft offensichtlich doch beschränkt. Man kann fragen, ob in diesem Diskurs diese Entwicklungen, Geschehensabläufe und Ereignisse richtig rekonstruiert werden, ob die Beobachtungen zutreffend sind, ob es tatsächlich Wissen ist oder Irrtum - das aber macht Singelnstein freilich nicht.

An anderer Stelle wird vom Leid im Gefängnis gesprochen (S. 100) - eine Kategorie, die im Übrigen nicht auftaucht. Mit der Festlegung auf Kriminalität als soziales Kon- 
strukt und der Orientierung an abweichendem Verhalten muss der Bezug auf Leid und Schädigung undeutlich bleiben, denn sonst würde die erkenntnistheoretische Position sich als tönern erweisen.

Insoweit kann sich Singelnstein von dem Diskurs distanzieren und gewinnt, entsprechend seinem Programm, tatsächlich Distanz. Doch fehlt das Instrumentarium, dies theoretisch zu internalisieren.

\section{Dialog}

$t s:$

Du hast eine sehr kritische Besprechung geschrieben. Dies ist nicht besonders überraschend, denn Du argumentierst aus einer grundsätzlich anderen Perspektive, als ich es in der Arbeit tue. Die foucaultsche Herangehensweise in der Diskursanalyse (DA) aber auch die Gouvernementalität sind in ihren Grundannahmen irritierend, da sie bestimmte Selbstverständlichkeiten und Prämissen bei der Betrachtung gesellschaftlicher Erscheinungen in Frage stellen oder gar umkehren und eine gewöhnungsbedürftige Terminologie mitbringen. Daher rufen sie schnell Abwehrreaktionen hervor, wenngleich sie sich in den Sozialwissenschaften zu einer breit aufgegriffenen Forschungsperspektive entwickelt und etabliert haben.

Ich begreife und nutze diese Sichtweise als eine Folie oder Brille, mit der sich kriminologische Fragestellungen auf eine bestimmte Art und Weise betrachten lassen. Ich behaupte nicht, dass sich damit alles ergründen ließe. Vielmehr bin ich der Auffassung, dass die DA ihre Berechtigung neben zahlreichen weiteren Ansätzen hat. Dieser Platz gebührt ihr meines Erachtens aber ohne Zweifel, denn sie ermöglicht wertvolle Einsichten, die anderen Ansätzen verschlossen bleiben.

Um dieses Verständnis, aber auch die Unterschiede in den Grundannahmen etwas darzustellen, ist es meines Erachtens hilfreich, drei Fragen aufzugreifen, die Du aufgeworfen hast:

- Ist der diskursanalytische Ansatz in sich schlüssig?

- Was kann er erklären bzw. wie weit reicht er?

- Wie ist das Verhältnis von Diskurs und materieller bzw. erfahrbarer Wirklichkeit?

Damit hängt Deine Behauptung zusammen, Beobachtungen und Handlungen brächen $d f$ : „außerdiskurslich“ ein, was der theoretische Rahmen nicht vorsehe.

Ja. Die Fragen hängen zusammen; wir sollten sie nicht abschnittweise, sondern verwoben abhandeln.

Wir haben uns beide auf den Korzybski-Satz „The map is not the territory“1 bezogen. Ich glaube, das ist ein guter Ausgangspunkt.

ts:

Man kann von einer Landschaft sehr unterschiedliche Landkarten malen, die aber nebeneinander ihre Berechtigung haben. Sie stellen die gleiche Landschaft unterschiedlich dar, wie etwa eine topografische und eine politische Karte. In diesem Sinne stellt die

1 Korzybski (1933): A Non-Aristotelian System and its Necessity for Rigour in Mathematics and Physics. Sciene and Sanity, S. 747-761. 
Diskursanalyse eine Karte bereit, die bestimmte Dinge sichtbar macht. Damit ist es ihr möglich, Zusammenhänge und Aspekte zu erhellen, die ansonsten unterbelichtet bleiben. Dafür blendet sie andere Aspekte aus. Man kann diesen Ansatz sehr fruchtbar für kriminologische Fragestellungen nutzen ${ }^{2}$ - alleine oder in Verbindung mit anderen Folien. Meines Erachtens ist es für das Verständnis einer Landschaft wichtig, verschiedene Landkarten parallel heranzuziehen.

$d f$ :

Genau. Aus diesem Grund fand ich Euer Buch zur „Sicherheitsgesellschaft“3 ${ }^{“ 3}$ sehr gut. Daher auch das Interesse für Deine Dissertation. Überrascht war ich, dass diese nicht an die frühere Arbeit anknüpft, sondern den alten Ansatz ersetzt und gerade nicht verschiedene Perspektiven kombiniert.

$t s$ :

Ich würde sagen, dass beide Herangehensweisen ihre Berechtigung haben. Man kann sich einem Gegenstand nähern, indem man ihn aus verschiedenen Perspektiven beleuchtet, wie wir es in der „Sicherheitsgesellschaft“ tun. Ebenso kann man aber auch einen theoretischen und methodischen Ansatz alleine konsequent verfolgen. Letzteres habe ich für die Dissertation getan, aus verschiedenen Gründen: Zum einen fand ich es reizvoll, den Ansatz einmal stringent für die Kriminologie anzuwenden und auszuprobieren. Zum anderen bedeutet eine Kombination immer auch eine Art Kompromiss. Das wollte ich vermeiden, sondern der DA in ihren Grundannahmen und mit der nötigen Tiefe nachgehen.

Dies bedeutet aber nicht, die theoretischen Grundannahmen dieses Ansatzes und insbesondere sein Verständnis von Wirklichkeit zum alleinigen Maßstab des eigenen Denkens zu machen und sich damit selbst der Möglichkeit zu entheben, „gesellschaftliche Realität jenseits eines Diskurses“" wahrzunehmen und einzubeziehen, Unwissen und Irrtum zu erkennen. Ebenso wenig bedeutet es ein Verbot, bestimmte Fragen zu stellen.

Eine Analyse, die die Verteilung der materiellen Ressourcen mit im Auge behält, wie Du es gefordert hast, ist sicher ebenso ein spannendes Unterfangen. Dass es sich dabei allerdings um die alleine ,zentralen Fragen“ handelt, möchte ich bezweifeln. Denn ohne interpretative Ebene und einen differenzierten machtanalytischen Ansatz droht auch eine solche Perspektive nur ,ein ,verschwindendes Moment“ im Kontext einer Kriminalitätstheorie“ zu sein. Dass Unwissen auf diesen Ebenen zumeist als weniger schmerzlich empfunden wird, mag auch damit zu tun haben, dass manche Realitäten offensichtlicher sind und eher einem herrschenden erkenntnistheoretischen Zugang entsprechen, als andere dies tun.

$d f$ :

Die Diskursanalyse nimmt sich die Analyse der Diskurse vor, wie in und durch Diskurse bestimmte Vorstellungen von Krankheit und ihrer Heilung, Kriminalität, ihren Ursachen und ihrer Bekämpfung entstehen, wie aus solchen Vorstellungen heraus Krankenhäuser,

2 Siehe dazu etwa Singelnstein (2010): Diskursives Wissen als Grammatik sozialer Kontrolle. Zur Rolle von Diskursen bei der Konstituierung von Abweichung und Kontrolle. In: Kriminologisches Journal, S. 115 ff.; Singelnstein/Ostermeier (2012): Wissenssoziologische Diskursanalyse in der Kriminologie. In: Keller/Truschkat (Hrsg.): Methodologie und Praxis der Wissenssoziologischen Diskursanalyse (im Erscheinen).

3 Singelnstein/Stolle (2012): Die Sicherheitsgesellschaft. Soziale Kontrolle im 21. Jahrhundert, 3. Aufl. 
Sicherungsverwahranstalten und Programme der Früherkennung entwickelt werden. Die „Landschaft der Diskursanalyse“ ist der Diskurs, das Hin und Her der Behauptungen, Argumente usw. Wenn die Diskursanalyse mir darüber Aufschluss verschafft, bin ich sehr froh: sie liefert mir eine Landkarte, mittels derer ich mich in dem untersuchten Diskurs orientieren kann.

$t s$ :

Ja, der DA zufolge sind Wahrnehmung und Handeln des Einzelnen durch Wissen geleitet, das in Diskursen gesellschaftlich produziert, legitimiert und transformiert wird. Solche Wissensbestände bestimmen, welche Bestandteile einer materiellen Wirklichkeit wir wahrnehmen und wie wir sie interpretieren. Verinnerlicht in Subjektivierungsprozessen prägt solches Wissen das Selbstverständnis des Einzelnen. Diskurse beschreiben nicht Realität, sondern sie konstituieren sie, indem sie Wissen, Bedeutung und Sinn über (bestimmte) Dinge hervorbringen, verbreiten, als wahr gelten lassen. Sie regeln, wo welches Wissen produziert, welche Themen bearbeitet werden und welche Perspektiven im Dunkeln bleiben. Dem Ansatz gelingt es damit, einerseits das Verhältnis von Praktiken und Wissen in einer konstruktivistischen Perspektive zu kombinieren und dabei andererseits gesellschaftstheoretische Aspekte zu integrieren.

Insofern passt auch der machtanalytische Ansatz der Gouvernementalität gut dazu. Dieser ist regelmäßig mit Einwänden konfrontiert, wie auch Du sie formuliert hast. Der Ansatz erhebt aber nicht den Anspruch, sämtliche gesellschaftlichen Machtverhältnisse abzubilden. Ihm geht es vielmehr darum, Formen von Macht analytisch zu erfassen, die bei einem herkömmlichen Verständnis von Macht und Herrschaft unterbelichtet bleiben. Das bedeutet nicht, dass es keine Macht und Herrschaft im klassischen Sinne gebe. Daher geht es ihm - und mir in meiner Arbeit - nicht darum zu ergründen, wer Gefängnisbauten bezahlt und warum. Gesucht wird vielmehr nach subtilen und produktiv wirkenden Formen der Macht, die die gesamte Gesellschaft auf sehr vielfältige Art und Weise durchziehen und jeden Einzelnen betreffen. Hiermit verbunden ist etwa die Frage nach der machtvollen Wirkung von gesellschaftlichen Interpretationen und Vorstellungen, die dazu führt, dass sich der Einzelne rational verhält - das heißt rational im Sinne der herrschenden Rationalität des Diskurses. Bei der Suche nach solchen Formen von Macht aber gibt es kein klares Oben und Unten, ist die Frage „Wer herrscht?“ nicht so einfach zu beantworten, lassen sich machtvolle Akteure oder Strukturen, nach denen eine kritische Perspektive sucht, nicht so klar herausschälen und als Gegenüber identifizieren. Dies mag man als blass, verschwommen oder unscharf empfinden.

$d f$ :

Ich bin einig mit Dir, dass es diffuse Machtprozesse gibt. Bestimmte Wirklichkeitsvorstellungen oder Modelle setzen sich durch, besetzen die Köpfe und bestimmen so Handlungen. Der Ansatz verspricht ein Raster oder Schema, welches die Wahrnehmung lenkt, wo und wie sich unterschiedliche und asymmetrische Einflussverhältnisse, die nach den klassischen Konzeptionen unterhalb eines Schwellenwerts lagen, zeigen und dann abbildbar machen. Was ich vermisst habe, war eine entsprechende Umsetzung, d.h. die Probe aufs Exempel, die Du für die DA lieferst.

Mein Einwand ist, dass Diskursanalytiker dazu neigen, die Analyse des Diskurses mit der Analyse der Gegenstände des untersuchten Diskurses gleichzusetzen, anzunehmen, dass mit der Analyse des Diskurses über gefährliche Straftäter etwas über gefährliche Straftäter ausgesagt wird, über ihre Existenz, über ihr Wesen. Der Diskurs selber bezieht 
sich auf die Landschaft und zeichnet eine Karte, in der gefährliche Straftäter vorkommen. Das garantiert nicht ihre Existenz. Das ist die Frage der Reichweite. Die DA kennt ihre Grenzen nicht.

ts:

Ich würde sagen: Hier besteht keine Grenze der DA, hier liegt ihr Clou. Die DA macht es sich ja nicht zur Aufgabe, bloß die Verläufe gesellschaftlicher Debatten über bestimmte Themen zu verfolgen. Sie will vielmehr erklären, wie die Landschaft, wie Dinge in der Gesellschaft entstehen und sich wandeln. Dabei kehrt sie, wenn Du so willst, die herkömmliche Perspektive, wie Du sie auch gerade beschrieben hast, um. Gesellschaftliches Wissen, also Diskurse, sind danach nicht Ergebnis gesellschaftlichen Redens über bestehende Dinge, sondern das Wissen geht den Dingen vor. Diese werden erst von den Subjekten in Praxen hervorgebracht, die auf Wissen basieren. Somit folgt nicht der Diskurs aus den Gegenständen, aus einer materiell existenten Wirklichkeit, sondern sind umgekehrt die Gegenstände Folge bestimmter Diskurse. Gefährliche Straftäter sind demnach keine gesellschaftliche Entität, sondern Produkt sozialer Praxen, die auf Wissen basieren, das auch anders sein könnte. In diesem Sinne stimmt auch der KorzybskiSatz nicht. Denn das Wissen ist eben nichts ganz anderes als die Wirklichkeit, die ja gerade aufgrund dieses Wissens hergestellt wird. Die Karte ist also kein Abbild einer bestehenden Wirklichkeit, sondern prägt unsere Wahrnehmung, unseren Umgang mit dieser. Die DA hat also ein besonderes Verständnis von Wirklichkeit, das zunächst irritiert, weil es im Widerspruch zu unserem herkömmlichen Verständnis steht. $d f$ :

Ich gehe hartnäckig davon aus, dass die „Landschaft“ der Kriminalwissenschaft - das Referenzobjekt - das Verbrechen ist. Eine erste Aufgabe dieser Wissenschaft ist es zu klären, ob es Verbrechen, Verbrecher, Schuld, Hang, gefährliche Straftäter gibt. Die Diskursanalyse (DA) sieht ihre Aufgabe darin, die Entstehung der Vorstellungen von all den genannten Entitäten zu beschreiben. Wenn ich mich vergewissert habe, dass es Verbrechen pp. gibt, kann es immer noch sein (und ist nur zu häufig so), dass meine Vorstellungen über das Verbrechen und sein Wirken und Wesen irrig sind.

Ich kann wissen, dass es eine Insel I gibt und doch eine Landkarte davon haben, die mich in einen Sumpf geraten lässt, den die Landkarte nicht verzeichnet. Dann, würde ich behaupten, ist die Landkarte falsch. Die DA fragt: Wie kamen die Kartographen A dahin, den Sumpf 5 km entfernt einzuzeichnen, die anderen Kartographen B, ihn als See zu vermerken, und C, die ganze Insel zu übersehen. Die Karte von D, die den Sumpf an der richtigen Stelle verzeichnet hatte, war aus dem Diskurs der Kartographen verschwunden, die anderen waren mächtiger. Ich sage: „Hätte ich nur die von D genommen, hätten mich die Retter nicht aus dem Sumpf ziehen müssen.“

ts:

Der DA geht es nicht darum, eine materiell existente, objektive Wirklichkeit zu ergründen oder zu beschreiben. Das ist einfach nicht ihre Frage. Sie ist auf der Suche nach den sich wandelnden Wissensbeständen, die unsere Interpretation und unser Handeln leiten, da diese aus ihrer Perspektive wesentlich bedeutsamer sind. Dementsprechend geht es ihr nicht um die Freilegung einer verdeckten Wahrheit oder Objektivität, um die Prüfung diskursiven Wissens auf Wahrheit, „Irrtum oder Unwissen“, sondern um die Darstellung der Kontingenz von Wahrheit und Wirklichkeit, das heißt zu zeigen, dass diese nicht festgelegt ist, sondern auch anders sein könnte, werden kann, bloße Möglichkeit ist. 
$d f$ :

Also gibt es nur Wissen, keine Wissenslücken, Unwissen, keinen Irrtum? Dies verbietet mir implizit, die Realitätsadäquanz der im Diskurs entworfenen Weltmodelle in Frage zu stellen. Wenn der Diskurs Wissen erzeugt und enthält, stehe ich mit meiner Behauptung „Ihr wisst es nicht“ gegenüber den Diskursführern blöd da. Die sagen nämlich: „Der Diskursanalytiker hat unser Wissen bestätigt.“

$t s$ :

In der Tat, da es in der Perspektive der DA keine objektive, erfahrbare Wahrheit gibt, die nur durch die „falsche“ Wahrheit des Diskurses verdeckt würde. Materielle Wirklichkeit lässt sich in dieser Perspektive nicht von sinnhafter Zuschreibung trennen und befreit von dieser darstellen. Unsere Wirklichkeit ist immer bereits eine Interpretation der Materialität, sodass es eine „tatsächliche“, objektive Wahrheit nicht gibt. Die DA berücksichtigt das, was gemeinhin als Wirklichkeit angesehen wird, damit quasi nur mittelbar, wenn sie davon ausgeht, dass Gegenstände, Handlungen und Ereignisse nicht objektiv, unmittelbar wahrgenommen werden, sondern diese Wahrnehmung stets schon durch das Wissen geprägt ist, das unser Denken und Handeln leitet.

Daher ist auch das Erkenntnisinteresse dieses Ansatzes eine anderes. Es geht der DA wie gesagt nicht darum zu ermitteln, ob ein bestimmtes Wissen richtig oder falsch ist. Sie untersucht ,nur“ die Entstehung und den Wandel von Wissensbeständen, die zu einer bestimmten Zeit gültig - und daher im Sinne der DA wahr - sind. Es geht also nicht um die differierende Interpretation einer bestehenden Wirklichkeit, sondern um die Untersuchung von deren wissensbasierter Hervorbringung durch die Praktiken der Subjekte.

$d f$ :

Gibt es unwissensbasierte Hervorbringungen?

ts:

Ich würde sagen nein: jede Hervorbringung von Wirklichkeit durch Handeln basiert jedenfalls auch auf Wissen.

$d f$ :

„Wissensordnungen“ ist ein ,beschlagnahmender" Terminus. Rückblickend sind viele vermeintliche Wissensordnungen als mit hohem Unwissensgrad erkannt worden. Der Ansatz baut die Vorstellung eines perpetuum mobile.

Begriffe wie Wissen, Wahrheit und Wirklichkeit belegt die DA mit Beschlag. Ihnen wird eine eigentümliche Bedeutung zugeschrieben, die die Analyse hermetisch abschließt. Wenn man einen Dialog über die Adäquanz bestimmter Ansätze führen will, braucht man einen gemeinsamen Wortschatz, d.h. bestimmten Namen müssen übereinstimmend sprachliche Definitionen zugeordnet werden, so dass man damit auf denselben Gegenstand verweist. Wenn Wissen so wie von Dir definiert wird, zieht mir das den Boden unter den Füßen weg. Daher kamen meine drastischen Bemerkungen über den „Fluch der Geisteswissenschaft“, zumal ich überrascht war, dass Du diesen Ansatz im Buch ohne Fragezeichen und „Rahmung“ vertrittst. Ich mache mein Problem mit folgenden Beispielen deutlich:

„Rechts ist der Lichtschalter. Ich weiß es genau.“ Alle Diskursteilnehmer stimmen zu. Der Sprecher betätigt den Schalter. Der Scheibenwischer geht an.

„Aderlass ist das Mittel der Wahl bei dieser Krankheit.“ Patient stirbt. Medicus: nicht genug Blut abgelassen. Mache ich beim nächsten Mal besser. 
„X ist eine Hexe. Ich habe im Hexenhammer nachgelesen. Der Richter hat es auch festgestellt. Hexen gehören verbrannt, weil sie gefährlich sind und um ihres Seelenheils willen." Allgemeine Zustimmung. Alle ,wissen", dass es Hexen gibt. X wird als Hexe verbrannt.

Y wird als gefährlicher Straftäter angesehen und dementsprechend behandelt. Infolge der Behandlung begeht er weitere erhebliche Straftaten. „Wir haben es doch gleich gewusst".

In allen Fällen behaupte ich: Unwissen, Irrtum. Gleich, wie viele, wie tief überzeugt sind von dem, was sie sich vorstellen. Selbst wenn die Ausgangsbehauptung, wie in (4), in einer selffulfilling prophecy scheinbar validiert wird. Dazu kommt es aber nur, weil man die Zeit vergisst.

$t s$ :

Ich finde, gerade Aderlass und Hexenverbrennung sind sehr gute Beispiele für die Rolle diskursiven Wissens. Es war zu deren Zeiten gültiges Wissen, dass Aderlass eine probate Heilbehandlung ist und dass es Hexen gibt, gegen die man etwas unternehmen muss. Das ist für uns heute Unsinn. Damals war es aber eben wahr und Grundlage für soziale Praxen in der Gesellschaft, die dieses Wissen stetig reproduziert und so Wirklichkeit hergestellt haben. Genau diese Zusammenhänge untersucht die DA. Welche der beiden Vorstellungen nun richtiger ist, spielt für sie eigentliche keine Rolle, zumal sie eben bezweifelt, dass es eine letztgültige Wahrheit gibt.

$d f$ :

Was in der DA als Wissen bezeichnet wird, ist Vorstellung, Glaube, Überzeugung. Glaube kann Berge versetzen, keine Frage. Aber zwischen (mehr oder minder) richtigen Vorstellungen, Wahn, Halluzination und Irrtum sollte man unterscheiden können und diesen Unterscheidungen auch unterschiedliche sprachliche Bezeichnungen zukommen lassen. Auch eine Massenhysterie oder ein kollektiver Wahn - alle verstärken sich diskursiv in ihren Überzeugungen - ist gewöhnlich von falschen Vorstellungen getragen. Keine Frage auch, dass wir die Wirklichkeit nie umfassend, nie vollkommen objektiv erfassen, dass unsere sprachlichen Möglichkeiten der Beschreibung die inneren Modelle modifizieren, verzerren können. Aber mit einem ptolemäischen Weltmodell kann man nicht zum Mond fliegen. Das macht es relativ falsch. Newton, ich glaube, war in diesem Punkt wirklich auf sich gestellt, drehte die Frage nach der Bewegung in die nach dem Stillstand. Das war ein zentraler Wendepunkt in der Mechanik. Für viele Zwecke genügte die vornewtonsche Mechanik. Wir sehen die Sonne auf- und untergehen und wissen doch, dass wir gedreht werden.

Ich kann viel mit der Diskursanalyse anfangen, aber nicht, wenn sie das A und O zu sein vorgibt.

$t s$ :

Ich sehe diesen von Dir gemachten Unterschied zwischen Wissen einerseits und Vorstellung andererseits so nicht. Das gleiche hätten Dir die Menschen im Mittelalter bzw. in der frühen Neuzeit mit der gleichen Inbrunst entgegengehalten, wenn Du ihre damals gültige Weltsicht in Frage gestellt hättest. Nur dass ihre Wahrheit eben gewesen wäre, dass es Hexen gibt und dass Aderlass ein gutes Heilmittel ist. Die von Dir als Wahrheit bezeichneten Dinge sind nichts anderes als Vorstellungen und Überzeugungen, die heute zwar gültig, letztlich aber kontingent sind. Diese Vorstellung bedeutet nicht, keinen „Begriff von Realität“ zu haben. Es ist nur ein anderer. 
Die Diskursanalyse negiert die Existenz einer materiellen Wirklichkeit nicht, hält sie nicht ,für irreal oder nicht feststellbar“. So ,übergriffig“, wie Du behauptest, ist die DA nicht. Aus ihrer Sicht spielt die materielle Wirklichkeit nur nicht die alles entscheidende Rolle, sondern ihre Wirkung und Bedeutung ist von den Interpretationen abhängig, die die Subjekte bei ihrer Wahrnehmung vornehmen. Das Wissen ist auch die Basis für Handlung und Interaktionen, die Dinge hervorbringen. Unterschiedliches Wissen bringt durch das Handeln der Subjekte unterschiedliche Realitäten hervor, wie sich etwa anhand der Kriminalitätstheorien der vergangenen Jahrhunderte und der durch sie hervorgebrachten Tätertypen und Kriminalitätsformen nachzeichnen ließe. Als Materialisierung lässt es sich angesichts dessen bezeichnen, wenn sich ein bestimmtes Wissen in festeren Formen niederschlägt - sei es als Recht in Form von Gesetzen, sei es als Gefängnis, Videokamera oder Intensivtäterdatei. Der Diskurs „,rekonstruiert“ daher gerade nicht „Entwicklungen, Geschehensabläufe und Ereignisse“, sondern bringt sie hervor durch das wissensbasierte Handeln der Subjekte. Das Wissen ist daher nicht richtig oder Irrtum, sondern einfach Grundlage des Handelns, das Wirklichkeit produziert.

$d f$ :

Weitgehende Zustimmung. Aber das „sondern“ trifft nicht zu. Den Hervorbringungen geht immer der Entwurf eines Weltmodells (für den jeweiligen „Weltausschnitt“) voraus - unter Einschluss der Einbettung von Wahrnehmungen in theoretische Vorstellungen, wie die Dinge zusammenhängen usw.

ts:

Ja, natürlich, aber genau dies ist doch durch das Wissen der Diskurse geprägt.

$d f$ :

Ich sehe keinen guten Grund, darauf zu verzichten, die Realitätsadäquanz solcher (vorgeblichen) Wissensbestände in Frage zu stellen.

ts:

Genau das tut doch die DA. Indem sie die Kontingenz dieser Wissensbestände herausarbeitet, stellt sie ihre Geltung in Frage. Anders als Du setzt sie nur keine ,wirkliche Wahrheit" entgegen, die sich vermeintlich hinter der Ideologie, dem falschen Wissen verbirgt. Denn für den Einzelnen ist die materiell existente Wirklichkeit nur durch das Wissen der Diskurse erkenn- und verstehbar, dass sich umgekehrt wiederum in bestimmten materiell existenten Dingen niederschlägt.

Dabei handelt es sich sicherlich um einen Kreislauf, da hast Du recht. Insofern ist das „sondern“ etwas überspitzt. Das liegt daran, dass diese zweite Wirkrichtung von Wissen und Diskursen meist unterbelichtet ist, sie nur als Abbild einer existenten Wirklichkeit angesehen werden.

$d f$ :

Diese Trennung von diskursiver Wahrheit und materieller Realität hält die DA aber selbst nicht durch. Wie in der Besprechung dargestellt, brechen Beobachtungen außerdiskurslich ein.

$t s$ :

Sicherlich gibt es - und darauf beziehst Du Dich ja auch im Kern - methodische Schwierigkeiten und Brüche bei der Umsetzung in empirische Forschungsprojekte. Insbesondere kommt eine diskursanalytische Methodik oft nur schwer ohne Bezug auf eine materielle Wirklichkeit aus. Ein solcher Bezug ist natürlich problematisch, wenn man davon ausgeht, dass die Wahrnehmung der Wirklichkeit stets diskursiv geprägt ist, so- 
dass eine davon befreite Feststellung von Wirklichkeit streng genommen nicht möglich ist. Ansonsten wäre man oft darauf beschränkt, den Wandel bestimmter Wissensbestände nachzuzeichnen, ohne dessen Wirkungen thematisieren zu können.

$d f$ :

Die Benennung dieses Problems in der Arbeit hätte mir als Leser sehr geholfen. Dann wären die in der Besprechung genannten ,außerdiskurslichen Einbrüche“ weniger einbruchhaft erschienen. Wie ich sehe, sind wir gar nicht so weit auseinander.

ts:

Diese methodischen Probleme sind zwar sicher nicht unerheblich. Dies ändert aber nichts daran, dass der Ansatz theoretisch gesehen in sich schlüssig ist, auch wenn er natürlich - wie jeder andere Ansatz auch - nicht alles erklären kann. Ebenso stellen die methodischen Probleme meines Erachtens keinen Grund dar, diesen innovativen Ansatz nicht auch für die Kriminologie zu verfolgen - zumal er noch relativ jung ist, sodass auf eine produktive Auseinandersetzung mit den Problemen gehofft werden kann. Dies gilt insbesondere, da er sich gut an bisherige Ansätze im gesellschaftskritischen Paradigma der Kriminologie anschließen lässt.

$d f$ :

Hoffentlich haben wir, durch Dissertation, Besprechung und diesen Dialog schon etwas $\mathrm{zu}$ dieser Auseinandersetzung beigetragen. 